\title{
Evolución microestructural durante el envejecimiento a alta temperatura de una aleación $35 \mathrm{Ni}-25 \mathrm{Cr}-\mathrm{Nb}$
}

\author{
Microstructure evolution during \\ high temperature aging of a \\ $35 \mathrm{Ni}-25 \mathrm{Cr}-\mathrm{Nb}$ alloy
}

Matías Sosa Lissarrague ${ }^{1,2}$, Fernando Prado ${ }^{2,3}$, Alberto Picasso ${ }^{1,4}$, Silvina Limandri ${ }^{2,5}$

\footnotetext{
${ }^{1}$ Universidad Nacional del Sur - Dpto. de Ingeniería, Av. L.N. Alem 1253, Bahía Blanca, Buenos Aires, Argentina. e-mail: mhs11986@gmail.com

${ }^{2}$ Consejo Nacional de Investigaciones Científicas y Técnicas (CONICET), Buenos Aires, Buenos Aires, Argentina.

${ }^{3}$ Universidad Nacional del Sur - Dpto. de Física, Bahía Blanca, Buenos Aires, Argentina.

${ }^{4}$ Comisión de Investigaciones Científicas de la Provincia de Buenas Aires (CICPBA), La Plata, Buenos Aires, Argentina.

${ }^{5}$ Universidad Nacional de Córdoba - Facultad de Matemática, Astronomía y Física (FAMAF), Córdoba, Córdoba, Argentina.

e-mail: fprado2007@gmail.com; alberto.picasso@uns.edu.ar; silvinalimandri@gmail.com
}

\section{RESUMEN}

La microestructura dendrítica de la aleación $35 \mathrm{Ni}-25 \mathrm{Cr}-\mathrm{Nb}$, en su condición as-cast, está constituida por una matriz austenítica y carburos eutécticos primarios de dos tipos: $\mathrm{MC}$ ricos en $\mathrm{Nb}$ y $\mathrm{M}_{23} \mathrm{C}_{6}$ ricos en $\mathrm{Cr}$ presentes en bordes interdendríticos y bordes de grano. Durante el envejecimiento a $800^{\circ} \mathrm{C}$ y diferentes tiempos, en la matriz precipitan carburos secundarios del tipo $\mathrm{M}_{23} \mathrm{C}_{6}$, mientras que los carburos del tipo MC, transformarían al siliciuro conocido como fase $\mathrm{G}\left(\mathrm{Ni}_{16} \mathrm{Nb}_{6} \mathrm{Si}_{7}\right)$. Se caracterizó la microestructura de la aleación mediante microscopía óptica, microscopía electrónica de barrido (SEM) con mapeo, difracción de electrones retrodispersados $(E B S D)$ y ensayos de dilatometría, con el objeto de detectar la presencia de esta fase indeseable y analizar las condiciones bajo las cuales se presenta. Se encontró Si en bordes de carburos primarios de $\mathrm{Nb}$. Esto podría estar señalando que, la transformación de este carburo hacia el siliciuro rico en $\mathrm{Ni}$ y $\mathrm{Nb}$, se encuentra en una etapa incipiente. Aparentemente, la transformación podría estar realizándose desde afuera hacia adentro del carburo de Nb. Además, la contracción observada en la curva de dilatometría, estaría asociada a la diferencia entre coeficientes de dilatación térmica entre la matriz y los carburos eutécticos primarios y no a la transformación del carburo $\mathrm{M}_{7} \mathrm{C}_{3}$ hacia el tipo $\mathrm{M}_{23} \mathrm{C}_{6}$.

Palabras clave: dilatometría, siliciuro rico en $\mathrm{Ni}-\mathrm{Nb}$, aleaciones resistentes a altas temperaturas.

\section{ABSTRACT}

The dendritic-type microstructure of the $35 \mathrm{Ni}-25 \mathrm{Cr}-\mathrm{Nb}$ alloy, in the as-cast condition, consists of an austenitic matrix and two types of eutectic primary carbides; $\mathrm{Nb}$-rich $\mathrm{MC}$ type and $\mathrm{Cr}$-rich $\mathrm{M}_{23} \mathrm{C}_{6}$ type both presents in interdendritic edges and grain boundaries. During aging at $800^{\circ} \mathrm{C}$ for different times, $\mathrm{M}_{23} \mathrm{C}_{6}$ type secondary carbides precipitate in the matrix while $\mathrm{MC}$ carbides would transform to a $\mathrm{Ni}-\mathrm{Nb}$ silicide, known as $\mathrm{G}$ phase $\left(\mathrm{Ni}_{16} \mathrm{Nb}_{6} \mathrm{Si}_{7}\right)$. The microstructure was characterized by optical microscopy, scanning electron microscopy (SEM) with mapping, electron backscatter diffraction (EBSD) and dilatometry, in order to detect the presence of this undesirable phase and analyze the conditions under which it is presented. Silicon was found on edges of primary Nb-rich carbides. This could be indicating that the transformation of this carbide towards silicide rich in $\mathrm{Ni}$ and $\mathrm{Nb}$, is in an incipient stage. Apparently, the transformation could be taking place from the outside to the inside of the $\mathrm{Nb}$ carbide. In addition, the contraction observed in the dilatometry curve would be associated to the difference on the thermal expansion coefficients between the matrix and the primary eutectic carbides and not to the transformation of the $\mathrm{M}_{7} \mathrm{C}_{3}$-type carbide to the $\mathrm{M}_{23} \mathrm{C}_{6}$-type.

Keywords: dilatometry, $\mathrm{Ni}-\mathrm{Nb}$ silicide, heat resistant alloys. 


\section{INTRODUCCIÓN}

La aleación HP modificada con $N b$ (35Ni-25Cr), es utilizada en la fabricación de tubos para hornos de pirólisis en la industria petroquímica. Por su alto contenido de níquel y cromo, estas aleaciones presentan una alta resistencia al creep y a la corrosión a temperaturas entre 650 y $1050^{\circ} \mathrm{C}$ [1-4]. La resistencia a la carburización, está determinada por la estabilidad de la austenita, la cual depende de la relación entre el contenido de cromo y de níquel. Altas concentraciones de estos elementos, retardan la difusión del carbono hacia el interior de la pared del tubo [5,6]. La microestructura as-cast está constituida por una matriz austenítica rica en $\mathrm{Ni}-\mathrm{Cr}-\mathrm{Fe}$; fortalecida por una red de carburos eutécticos primarios; los cuales, pueden ser de tipo $\mathrm{M}_{7} \mathrm{C}_{3}$ y/o $\mathrm{M}_{23} \mathrm{C}_{6}$ $(\mathrm{M}=\mathrm{Cr})$ y carburos $\mathrm{MC},(\mathrm{M}=\mathrm{Nb}, \mathrm{Ti})$. El Nb, en proporciones adecuadas, controla la precipitación de carburos secundarios, fragmentando más aún la red de los carburos primarios y retardando la coalescencia (coarsening) de los carburos secundarios, siendo éstos de menor tamaño [7]. Se ha señalado en la bibliografía que, la transformación del $\mathrm{NbC}$ a fases ricas en $\mathrm{Si}$ (siliciuros, $\mathrm{Ni}_{16} \mathrm{Nb}_{6} \mathrm{Si}_{7}$, fase $\mathrm{G}$ o $\mathrm{Ni}_{3} \mathrm{Nb}_{2} \mathrm{Si}$, fase $\eta$ ), es producida mediante un mecanismo de difusión donde el $\mathrm{C}$ es expulsado hacia la matriz, formándose de esta manera la precipitación secundaria. Estos siliciuros han sido identificados por otros autores en aleaciones similares a la analizada en el presente estudio, a temperaturas entre 700 y $1000^{\circ} \mathrm{C}$. Esta fase, de naturaleza predominantemente frágil, se forma en la interfaz matriz/carburos primarios de niobio [3,8,9]. Además, debido a que el proceso de difusión es más rápido en bordes de grano, la precipitación de la misma en la matriz, requiere de un tiempo mucho mayor. El objetivo del presente trabajo, fue caracterizar la microestructura inicial as-cast y analizar su evolución durante envejecimientos a $\mathrm{T}=800^{\circ} \mathrm{C}$ y diferentes tiempos.

\section{MATERIALES Y MÉTODOS}

A partir de un tubo de $100 \mathrm{~mm}$ de diámetro medio y $12 \mathrm{~mm}$ de espesor, se extrajeron muestras por corte de dimensiones 10x10x10mm, aproximadamente. Las mismas, fueron sometidas a un tratamiento térmico de envejecimiento en hornos resistivos y en atmósfera de aire a una temperatura de $800^{\circ} \mathrm{C}$, siendo luego extraídas a diferentes tiempos y enfriadas al aire. Posteriormente, fueron pulidas mecánicamente utilizando papeles abrasivos hasta grado 1200 y, finalmente, se realizó el pulido final utilizando alúmina de $3 \mu \mathrm{m}$. El ataque electrolítico de las muestras pulidas se realizó a temperatura ambiente, utilizando una solución acuosa de $\mathrm{KOH}$ al $10 \%$ bajo una tensión de $2 \mathrm{~V}$, durante $12 \mathrm{~s}$. En estas condiciones, las muestras pueden ser observadas mediante microscopía óptica y electrónica. Para tal fin, se empleó un microscopio óptico (MO) marca Leica con cámara digital y un microscopio electrónico de barrido (SEM) equipado con detector $E D S$ con mapeo, marca Zeiss modelo Sigma. Se utilizó un espectrómetro marca SpectroMax, para la determinación de la composición química de la aleación. El ensayo de dilatometría, se realizó mediante un dilatómetro de diseño vertical marca Linseis modelo L75VS1000C, a una temperatura constante de $800^{\circ} \mathrm{C}$; siendo la velocidad de calentamiento de la muestra de $2^{\circ} \mathrm{C} / \mathrm{min}$, hasta alcanzar dicha temperatura.

\section{RESULTADOS Y DISCUSIÓN}

En la Tabla 1, se presenta la composición química nominal del material analizado.

Tabla 1: Composición química de la aleación HP-Nb, expresada en \% en peso.

\begin{tabular}{|l|l|l|l|l|l|l|l|l|l|}
\hline \multirow{2}{*}{ ALEACIÓN } & \multicolumn{8}{|l|}{ COMPOSICION QUIMICA (\% P) } \\
\cline { 2 - 10 } & $\mathbf{C}$ & $\mathbf{S i}$ & $\mathbf{M n}$ & $\mathbf{C r}$ & $\mathbf{N i}$ & $\mathbf{N b}$ & $\mathbf{W}$ & $\mathbf{T i}$ & $\mathbf{F e}$ \\
\hline HP-Nb & 0,57 & 2,73 & 0,76 & 23,74 & 37,6 & 1,26 & 0,195 & 0,077 & Bal. \\
\hline
\end{tabular}




\subsection{Microestructura as-cast}

En la Figura 1, se muestra la microestructura de tipo dendrítica de la aleación en su condición as-cast, observada mediante microscopía óptica para bajo y alto aumento.
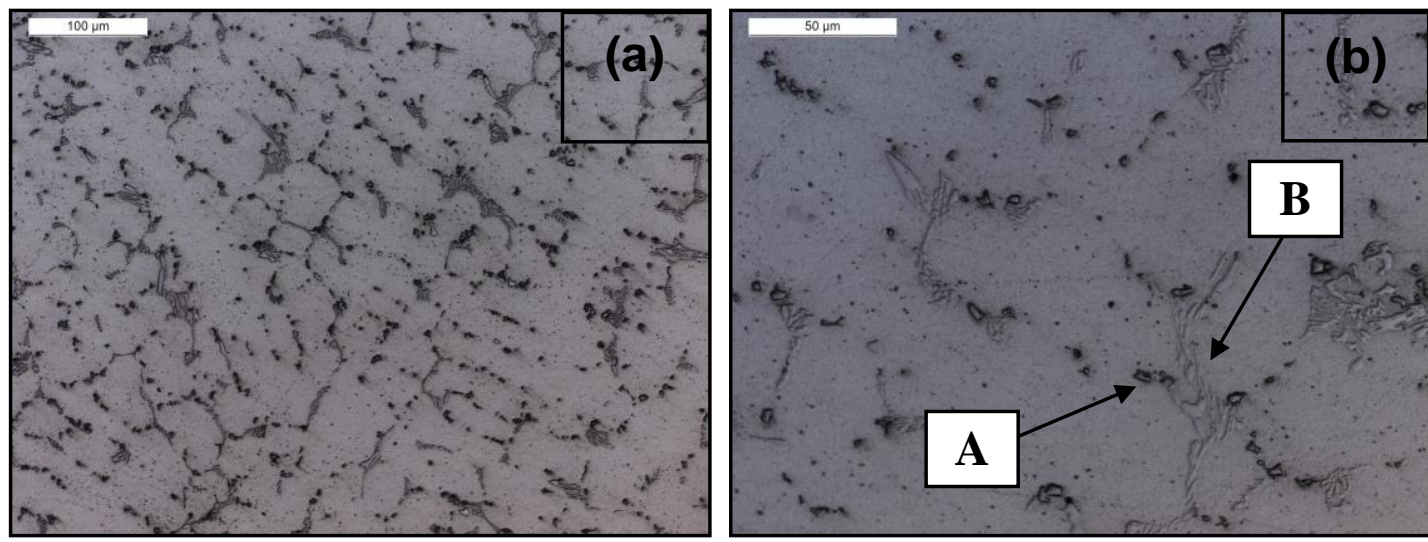

Figura 1: Micrografía óptica de la microestructura as-cast: a) 200X, b) 500X.

Como puede apreciarse en la Figura $1 \mathrm{~b}$, se distinguen dos tipos de partículas en bordes de grano y bordes interdendríticos: unas de tonalidad oscura (A) y otras de tonalidad clara (B). Estas últimas, presentan una morfología esquelética típica de los compuestos eutécticos. Además, se observan precipitados intragranulares e interdendríticos.

Con el fin de identificar las fases presentes en la microestructura as-cast, se obtuvieron micrografías $S E M$ en el modo de electrones retrodispersados (BSE), tal como se muestra en la Figura 2.

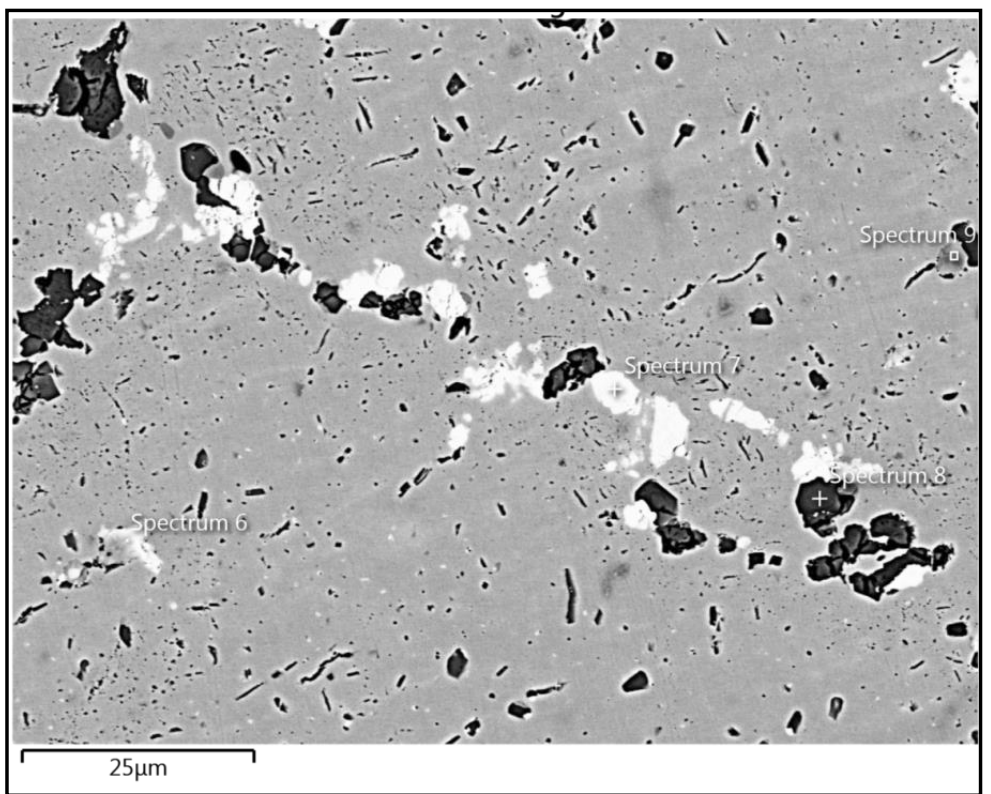

Figura 2: Micrografía SEM de la microestrctura as-cast.

De este modo, es posible lograr un contraste entre ambas fases, debido a la diferencia en el número atómico de los distintos elementos de mayor concentración, presentes en cada una de ellas. Es interesante destacar que las fases claras que aparecen en la micrografía de la Figura 2, se corresponden con las fases oscuras observadas en la micrografía óptica de la Figura 1b; mientras que, las fases oscuras que se observan en esta última se corresponden con las fases claras que aparecen, también en la Figura 1b. Por otro lado, se observan precipitados oscuros, en su mayoría con morfología acicular, en el interior de las dendritas y de los granos. Con el objeto de identificar estos compuestos, se realizó un mapeo de los elementos químicos en di- 
ferentes zonas de la muestra (ver Figura 2). De este modo y tal como se observa en la Figura 3, se pudo determinar la presencia y ubicación de los elementos; tales como, C, $\mathrm{Si}, \mathrm{Cr}, \mathrm{Fe}, \mathrm{Nb}$ y $\mathrm{Ni}$.
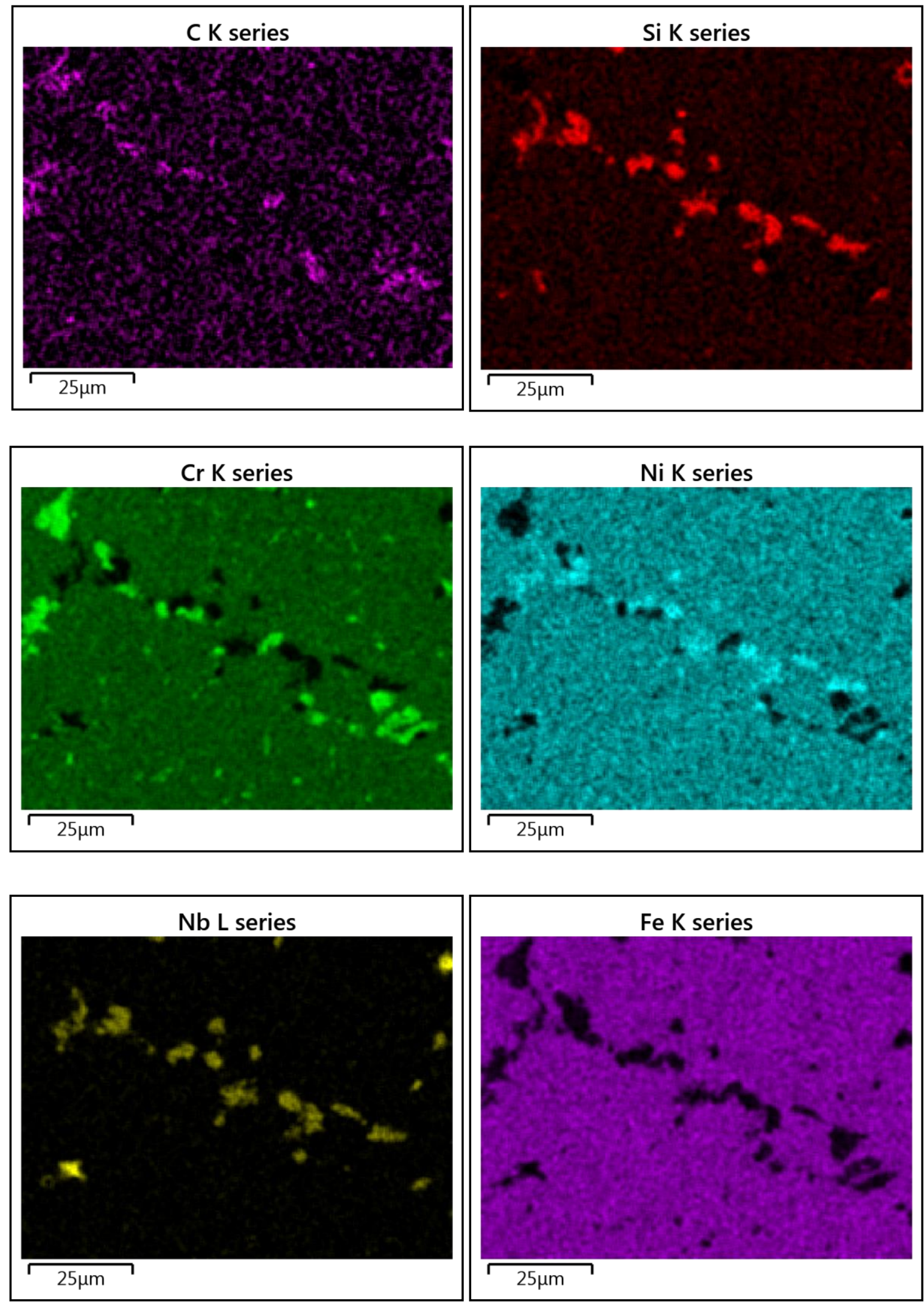

Figura 3: Mapeo de elementos químicos realizado en la microestructura as-cast. 
De acuerdo con los datos experimentales obtenidos mediante el mapeo de los elementos, podemos señalar que, los compuestos oscuros (ver Figura 2), ubicados en bordes interdendríticos, bordes de grano y dentro de las dendritas, son compuesto ricos en $\mathrm{C}$ y Cr. Por otra parte, los compuestos que se observan con tonalidad clara, son ricos en $\mathrm{C}$ y $\mathrm{Nb}$. Además, se observa que los precipitados en la matriz también presentan una alta concentración de $\mathrm{C}$ y $\mathrm{Cr}$.

En la Figura 4, se presenta una región de la microestructura a mayor aumento obtenida mediante SEM, donde puede observarse una fase de tonalidad clara. Para identificar su composición química, se efectuaron microanálisis $E D S$ en diferentes zonas de la misma y así, determinar su composición química semicuantitativa (ver Tabla 2). Puede observarse que, las concentraciones determinadas están en buen acuerdo con aquellos resultados obtenidos mediante mapeo; esto es, las fases claras muestran altas concentraciones de $\mathrm{C}$ y $\mathrm{Nb}$.

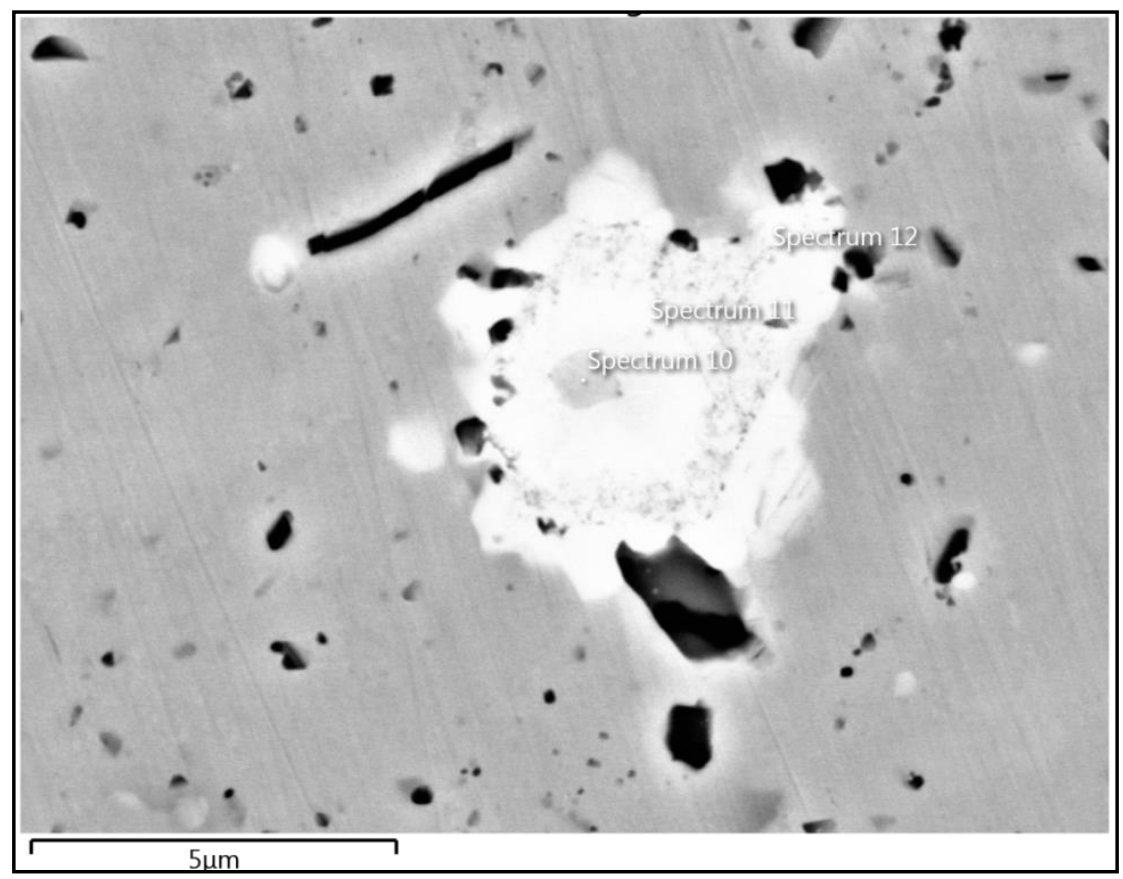

Figura 4: Micrografía SEM de una partícula de tonalidad clara.

Tabla 2: Resultado del microanálisis EDS en un compuesto rico en $\mathrm{C}, \mathrm{Nb}$ y Ti.

\begin{tabular}{|l|l|l|l|l|l|l|l|l|l|l|}
\hline PORCENTAJE (\%AT) & \multicolumn{1}{|l|}{ (\% } & $\mathbf{C}$ & $\mathbf{S i}$ & $\mathbf{M n}$ & $\mathbf{V}$ & $\mathbf{C r}$ & $\mathbf{F e}$ & $\mathbf{N i}$ & $\mathbf{Z r}$ & $\mathbf{N b}$ \\
\hline ELEMENTO & 44,7 & 13,5 & --- & --- & 0,58 & 2,95 & 1,16 & 2,76 & 4,59 & 13,5 \\
\hline Espectro 11 & 23,66 & 0,36 & 17,32 & 0,25 & --- & 3,75 & 5,84 & 36,67 & --- & 12,16 \\
\hline Espectro 12 & 0,70 & 0,12 & 0,03 & 0,03 & 0,04 & 0,05 & 0,04 & 0,06 & 0,13 & 0,34 \\
\hline Desvío estándar (\%) &
\end{tabular}

Una determinación por microanálisis realizada en una zona cercana al borde de la partícula (espectro 12), reveló la presencia de una alta concentración de $\mathrm{Si}$; lo cual, coincide con lo observado en el mapeo de elementos, analizado precedentemente. Estos resultados, están en acuerdo con aquellos señalados por otros autores [10-11]. En forma análoga, se realizó un microanálisis mediante EDS sobre una partícula oscura ubicada en un borde interdendrítico (espectro 14); tal como, se aprecia en la Figura 5. 


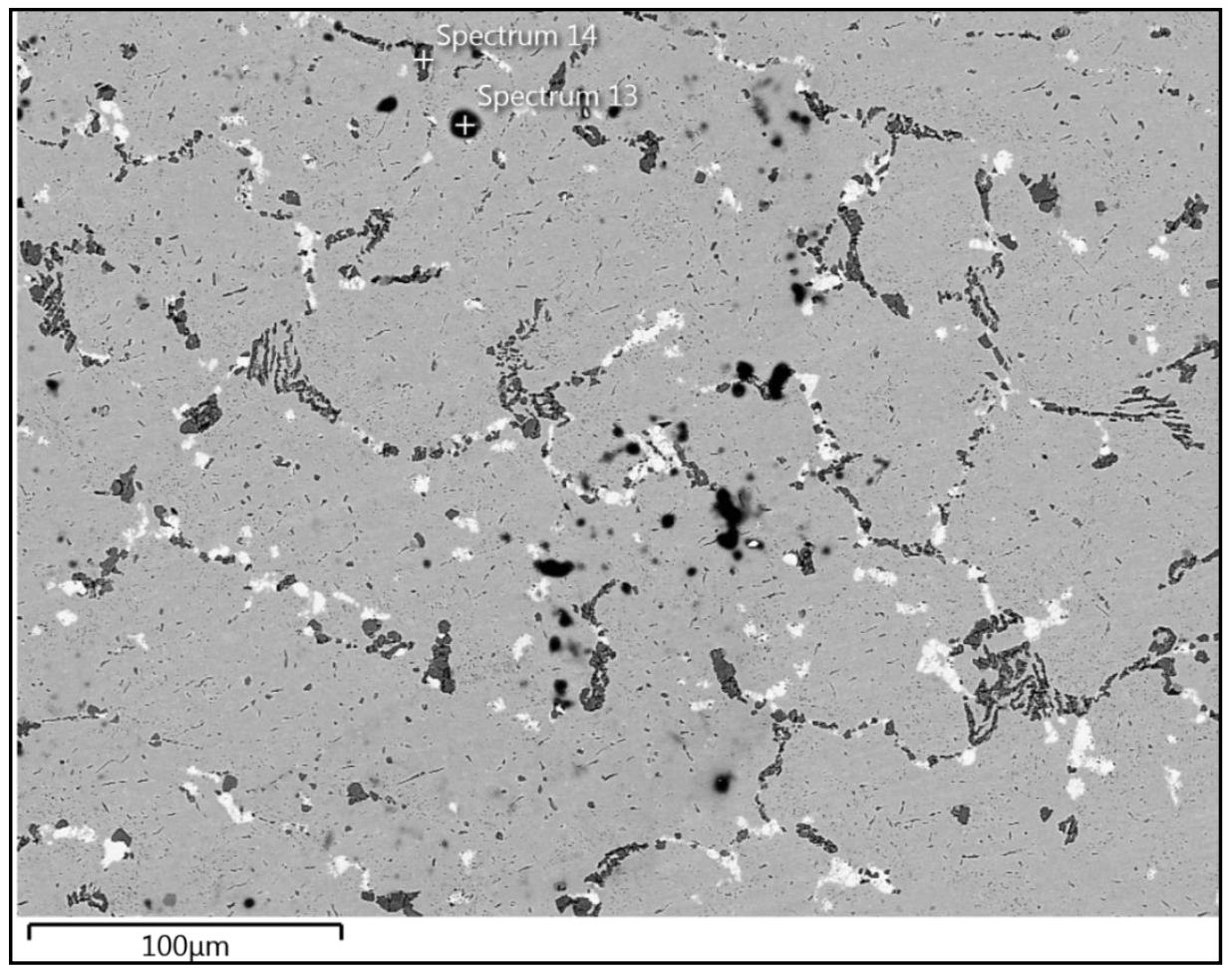

Figura 5: Micrografía SEM mostrando el punto de lectura del espectro EDS.

Su composición química semicuantitativa, es mostrada en la Tabla 3, de donde se desprende que esta fase es rica en $\mathrm{C}$ y en $\mathrm{Cr}$.

Tabla 3: Resultado del microanálisis $E D S$ en una fase rica en $\mathrm{C}$ y $\mathrm{Cr}$.

\begin{tabular}{|l|l|l|l|l|}
\hline PORCENTAJE (\%AT) \\
\hline ELEMENTO & $\mathbf{C}$ & $\mathbf{C r}$ & $\mathbf{F e}$ & $\mathbf{N i}$ \\
\hline Espectro 14 & 38 & 53 & 6 & 3 \\
\hline Desvío estándar (\%) & 0,30 & 0,30 & 0,10 & 0,10 \\
\hline
\end{tabular}

A partir de los resultados obtenidos podemos confirmar que, la red de carburos eutécticos primarios está compuesta por carburos ricos en $\mathrm{Cr}$ y carburos ricos en $\mathrm{Nb}$; mientras que, los precipitados intragranulares e interdendríticos son carburos ricos en $\mathrm{Cr}$. Sin embargo, las técnicas que hemos empleado hasta el momento, no nos permiten determinar con certeza la composición de las fases presentes en la microestructura inicial. Con el objeto de asegurar la identificación de estos compuestos en la condición as-cast, se recurrió a la técnica EBSD (Electron Back-Scatter Diffraction). En la Figura 6, se presentan los resultados.
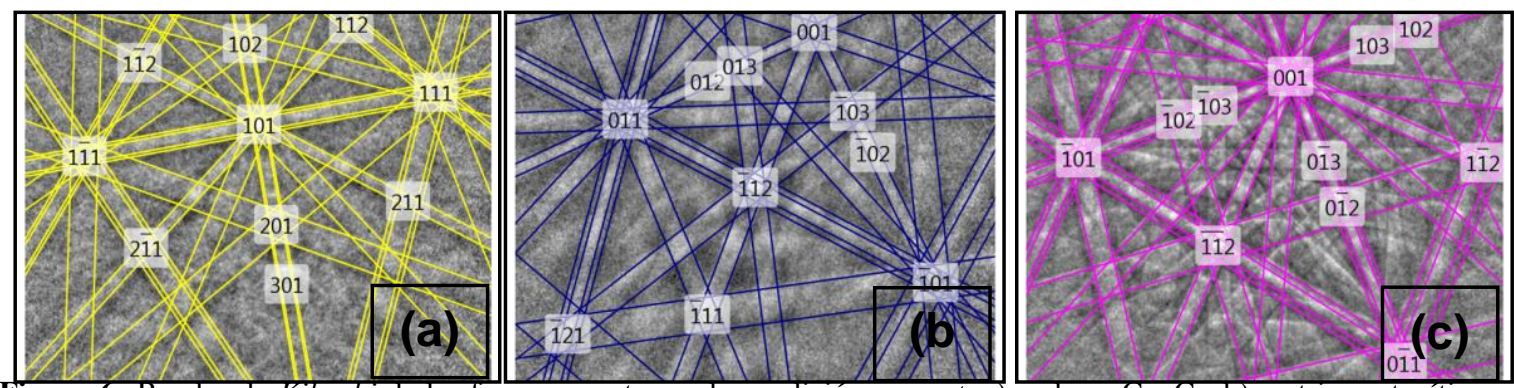

Figura 6: Bandas de Kikuchi de las fases presentes en la condición as-cast: a) carburo $\mathrm{Cr}_{23} \mathrm{C}_{6}$, b) matriz austenítica, c) carburo $\mathrm{NbC}$. 
En la Figura 6, las bandas de Kikuchi indexadas en color amarillo corresponden al carburo de Cr de tipo $\mathrm{Cr}_{23} \mathrm{C}_{6}$, aquellas indexadas en color azul pertenecen a la matriz austenítica y, finalmente, las bandas de color rosa, corresponden al carburo de $\mathrm{Nb}(\mathrm{NbC})$. Es importante destacar que, no se detectó la presencia del carburo primario tipo $\mathrm{M}_{7} \mathrm{C}_{3}$ bajo ninguna de sus estructuras cristalinas posibles, sea ésta hexagonal $\mathrm{u}$ ortorrómbica.

Por lo tanto, podemos confirmar que la microestructura en la condición as-cast de la aleación HP-Nb analizada en este trabajo, está compuesta de una matriz austenítica con una red de carburos eutécticos primarios ricos en $\mathrm{Nb}$ del tipo $\mathrm{NbC}$ y carburos ricos en $\mathrm{Cr}$ de tipo $\mathrm{M}_{23} \mathrm{C}_{6}$.

\subsection{Microestructura de muestras envejecidas}

En la Figura 7, se muestran la microestructuras de probetas envejecidas a $800^{\circ} \mathrm{C}$ y diferentes tiempos, observadas mediante microscopía óptica. Es posible señalar que, los carburos eutécticos primarios presentan una morfología distinta a la encontrada en la condición as-cast; además, se observa una zona libre de precipitados secundarios cercana a los carburos primarios (señalado por una flecha). Por otra parte, cabe destacar la presencia de precipitados secundarios de tipo agujas (ver Figura 7c).
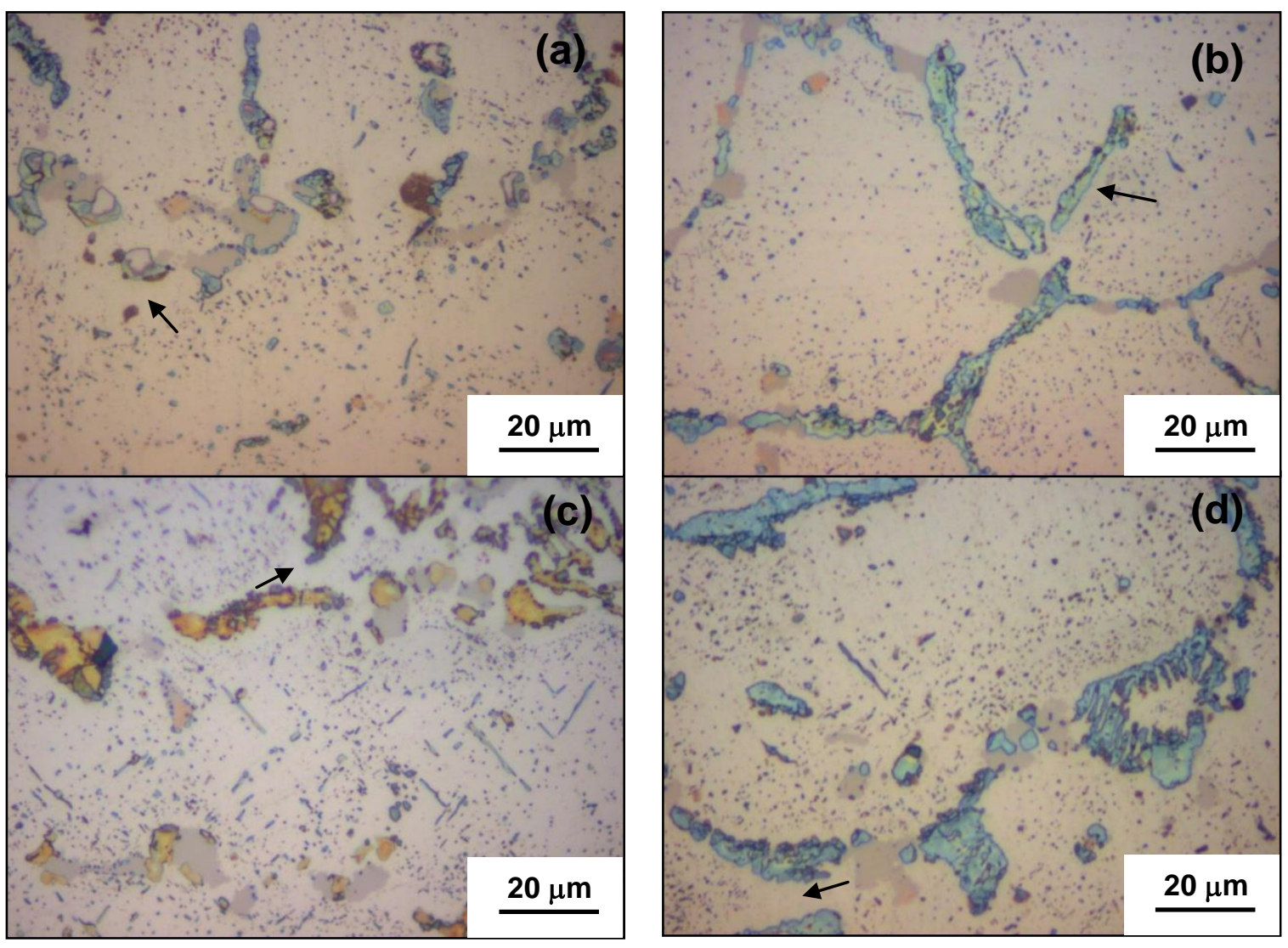

Figura 7: Imágenes de microscopía óptica de muestras envejecidas a $800^{\circ} \mathrm{C}$ para a) $16 \mathrm{~h}$, b) $72 \mathrm{~h}$, c) $143 \mathrm{~h}$ y d) $648 \mathrm{~h}$ $(1000 \mathrm{X})$.

En la Figura 8, se indican sobre una micrografía SEM las regiones donde se obtuvieron los espectros $E D S$ para la muestra envejecida a $800^{\circ} \mathrm{C}$ durante $143 \mathrm{~h}$. 


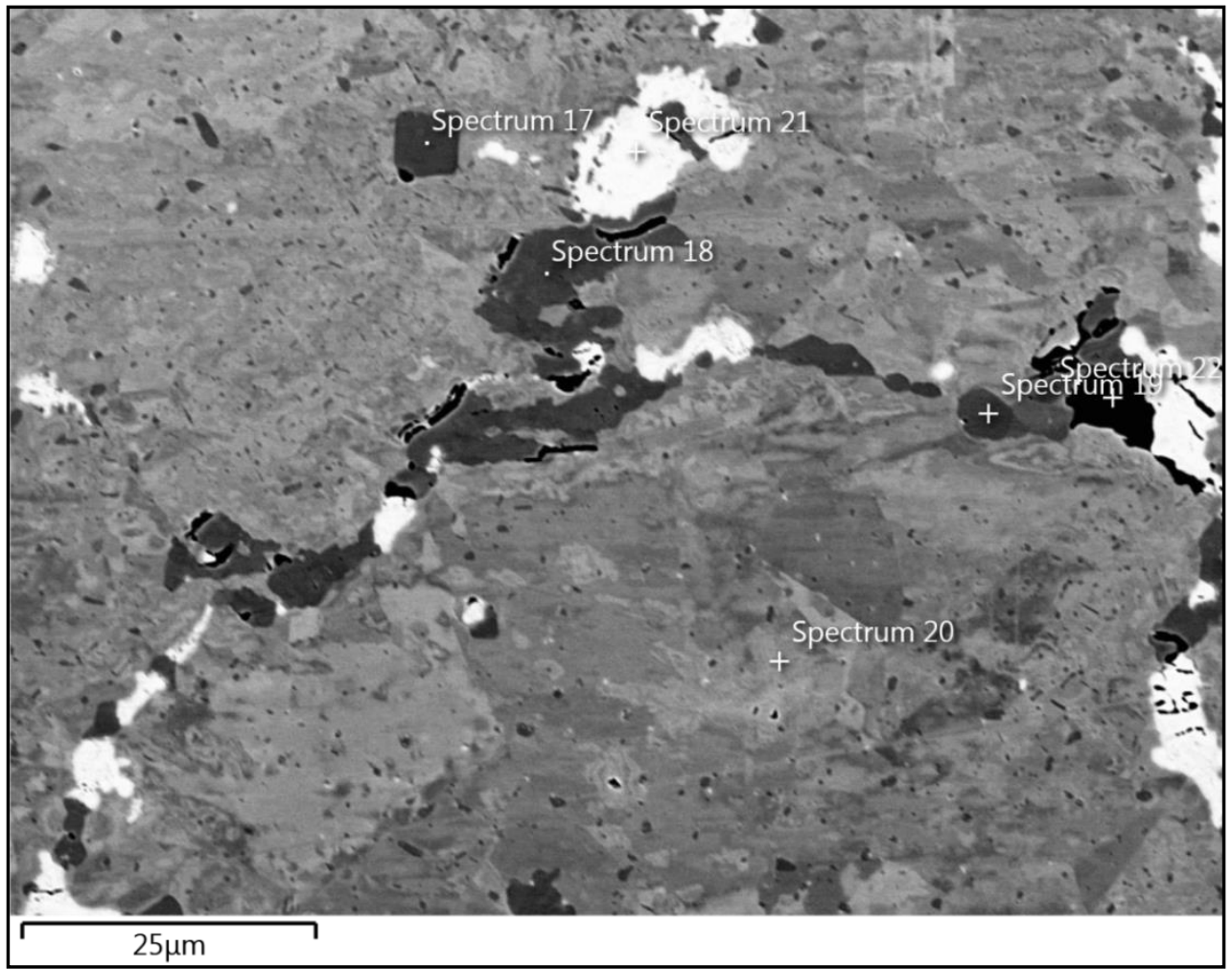

Figura 8: Puntos de lectura de espectros $E D S$ para muestra envejecida $800^{\circ} \mathrm{C}$ durante $143 \mathrm{~h}$.

Algunas de las composiciones químicas promedio determinadas sobre las partículas oscuras, ricas en $\mathrm{Cr}$ de la figura anterior, son presentadas en la Tabla 4 (espectros 17, 18 y 19). Es posible observar, que las partículas claras ubicadas en bordes de grano, presentan una composición química muy similar al compuesto $\mathrm{Ni}_{16} \mathrm{Nb}_{6} \mathrm{Si}_{7}$, conocido como fase $\mathrm{G}$ (espectro 21 ).

Tabla 4: Resultado del microanálisis $E D S$ en fases presentes en una muestra envejecida a $800^{\circ} \mathrm{C}$ durante $143 \mathrm{~h}$.

\begin{tabular}{|l|l|l|l|l|l|l|l|}
\hline ESPECTRO & $\mathbf{S i}$ & $\mathbf{T i}$ & $\mathbf{N b}$ & $\mathbf{C}$ & $\mathbf{C r}$ & $\mathbf{F e}$ & $\mathbf{N i}$ \\
\hline $\mathbf{1 7}$ & - & - & - & 24,14 & 67,51 & 4,98 & 3,37 \\
\hline $\mathbf{1 8}$ & - & - & - & 23,66 & 68,19 & 4,97 & 3,18 \\
\hline $\mathbf{1 9}$ & - & - & - & 23,87 & 67,78 & 5,13 & 3,22 \\
\hline PROMEDIO \%AT. & - & - & - & 23,89 & 67,83 & 5,02 & 3,26 \\
\hline $\mathbf{2 1}$ & 23,53 & 1,78 & 19,32 & - & 2,52 & 4,05 & 48,8 \\
\hline DESVíO ESTÁNDAR (\%) & 0,30 & 1,10 & 0,60 & 0,40 & 0,50 & 0,30 & 0,20 \\
\hline
\end{tabular}

Por otro lado, las determinaciones de composición química obtenidas sobre las partículas oscuras en bordes de grano, nos permiten afirmar que las mismas corresponden a carburos de Cr. Sin embargo, estos resultados no nos permiten diferenciar si el carburo es del tipo $M_{7} C_{3}$ o $M_{23} C_{6}$. De esta manera, en función de los resultados precedentes, resulta poco probable que estos carburos sean del tipo $\mathrm{M}_{7} \mathrm{C}_{3}$; ya que, en la condición as-cast no fueron encontrados.

Con el fin de investigar alguna probable transformación de fase durante el envejecimiento a temperatura constante, se llevaron a cabo ensayos de dilatometría en muestras as-cast. Tal como puede observarse en la Figura 9, una vez alcanzada la temperatura de $800^{\circ} \mathrm{C}$, vemos que se produce una contracción. 


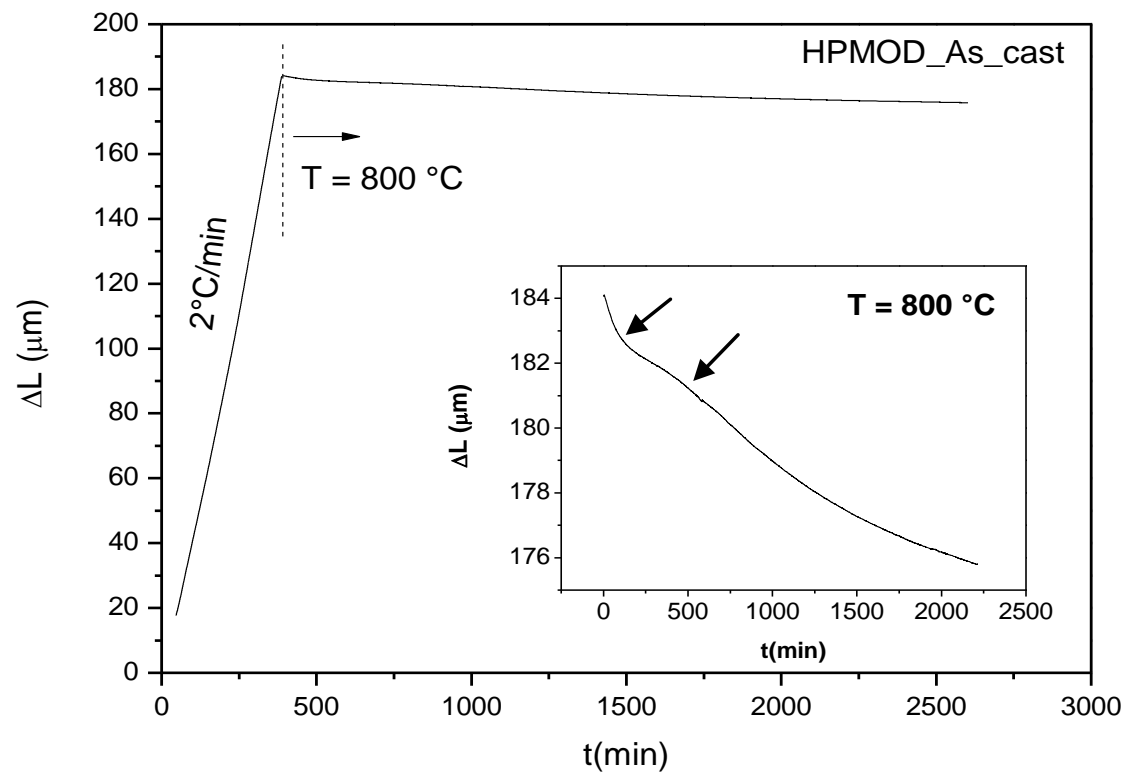

Figura 9: Curva de variación de longitud en función del tiempo, para una temperatura de $800^{\circ} \mathrm{C}$.

La contracción observada, puede explicarse a partir de la diferencia en los coeficientes de dilatación de la red de carburos eutécticos primarios y la matriz austenítica. A esta temperatura, la matriz tiene una expansión mayor a la soportada por los carburos primarios y de esta manera, existe un reacomodamiento en la interfaz que da lugar a dicha contracción [12].

Es interesante observar en la Figura 9 que, luego de un tiempo de ensayo isotérmico de alrededor de 50h, se observa un leve cambio en la velocidad de contracción de la muestra (región señalada entre flechas). Este comportamiento, podría estar asociado a la transformación experimentada por el carburo primario de tipo $\mathrm{MC}$ rico en $\mathrm{Nb}$, hacia el siliciuro rico en $\mathrm{Ni}$ y $\mathrm{Nb}$, el cual podría ser fase $\eta\left(\mathrm{Ni}_{3} \mathrm{Nb}_{2} \mathrm{Si}\right)$ ó fase $\mathrm{G}$ $\left(\mathrm{Ni}_{16} \mathrm{Nb}_{6} \mathrm{Si}_{7}\right)$. Por otra parte, es sabido que el C liberado en la transformación facilitaría la precipitación de carburos secundarios de tipo $\mathrm{M}_{23} \mathrm{C}_{6}$ en la matriz. Es probable que, la transformación del carburo MC conduzca a un aumento en el volumen; ya que, la red cristalina cúbica de la fase $\mathrm{G}$ y la fase $\eta$, tienen un parámetro de red casi dos veces y media mayor a la red cristalina del carburo NbC. Algunos autores [8], han encontrado la fase $\mathrm{G}$ en bordes de grano, luego de $2 \mathrm{~h}$ de envejecimiento en aleaciones muy similares a la estudiada $\mathrm{y}$ para la misma temperatura de envejecimiento. Sin embargo, es necesario diseñar nuevos experimentos para separar estas dos contribuciones.

\section{CONCLUSIONES}

Basados en los resultados obtenidos sobre el análisis en la condición as-cast y de envejecimiento en la aleación HP-Nb, es posible arribar a las siguientes conclusiones:

La microestructura de tipo dendrítica de la aleación HP-Nb en la condición as-cast, está formada por una matriz austenítica, fortalecida por una red de carburos eutécticos primarios ricos en $\mathrm{Nb}$ de tipo $\mathrm{NbC}$ y ricos en $\mathrm{Cr}$, del tipo $\mathrm{M}_{23} \mathrm{C}_{6}$. Se observan precipitados ricos en $\mathrm{Cr}$ en la matriz.

En la condición as-cast, se encontró $\mathrm{Si}$ en bordes de carburos primarios de $\mathrm{Nb}$. Esto podría estar señalando que, la transformación de este carburo hacia el siliciuro rico en $\mathrm{Ni} \mathrm{y} \mathrm{Nb}$, se encuentra en una etapa incipiente. Aparentemente, la transformación podría estar realizándose desde afuera hacia adentro del carburo de $\mathrm{Nb}$.

La contracción observada en la curva de dilatometría, estaría asociada a la diferencia entre coeficientes de dilatación térmica entre la matriz y los carburos eutécticos primarios y no a la transformación del carburo $\mathrm{M}_{7} \mathrm{C}_{3}$ hacia el tipo $\mathrm{M}_{23} \mathrm{C}_{6}$. 


\section{AGRADECIMIENTOS}

Los autores agradecen al Consejo Nacional de Investigaciones Científicas y Técnicas (CONICET), a la Comisión de Investigaciones Científicas de la Prov. de Bs. As. (CICPBA), a la Universidad Nacional del Sur, así como también a la Universidad Nacional de Córdoba.

\section{BIBLIOGRAFÍA}

[1] SHI, S., LIPPOLD, J., "Microstructure evolution during service exposure of two cast, heat-resisting stainless steels - HP-Nb modified and 20-32Nb", Materials Characterization, v. 59, pp. 1029-1040, 2008.

[2] GARBIAK, M., CHYLIŃSKA, R., "Precipitation kinetics in austenitic $18 \mathrm{Cr}-30 \mathrm{Ni}-\mathrm{Nb}$ cast steel", $\mathrm{Ar}$ chives of Foundry Engineering, v. 8, pp. 27-30, 2008.

[3] MOSTAFAEI, M., SHAMANIAN, M., Saatchi, A., et al., "Effect of aging temperature on structural evolution of HP-Nb heat resistant steel", International Journal of Iron \& Steel Society of Iran, v. 6, pp. 30-33, 2009.

[4] WANG, M., CHIU, Y., JONES, I., et al., "Microstructural characterization and microanalysis of creep resistant steels", Journal of Physics: Conference Series, v. 522, 2014.

[5] BAGNOLI, D., KRUPOWICZ, J., "Experiences with ethylene heater tube carburization", Corrosion/92, paper 307, 1992.

[6] BAIRAMOV, A., BRADLEY, R., "Local heavy carburization of olefin pyrolysis furnace tube", Corrosion 2002, paper 1389, 2002.

[7] YAN, J., GU, Y., DANG, Y., et al., "Effect of carbon on the microstructure evolution and mechanical properties of low Si-containing centrifugal casting 20Cr32Ni1Nb", Materials chemistry and Physics, IN PRESS, 2016.

[8] POWELL, D., PILKINGTON, R., MILLER, D., "The precipitation characteristics of 20\% Cr/25\% Ni-Nb stabilised stainless steel”, Acta Metallurgica, v. 36, pp. 713-724, 1988.

[9] SOARES, G. D.A., ALMEIDA, L. H., SILVEIRA, T. L., et al., "Niobium Additions in HP HeatResistant Cast Stainless Steels", Materials Characterization, v. 29, pp. 387-396, 1992.

[10] LAIGO, J., CHRISTIEN, F., LE GALL, R., et al., "SEM, EDS, EPMA-WDS and EBSD characterization of carbides in HP type heat resistant alloys", Materials Characterization, v. 59, pp. 1580-1586, 2008.

[11] GARBIAK, M., PIEKARSKI, B., "Phases in austenitic cast steel", Defect and Diffusion Forum Vols., pp. 215-220, 2012.

[12] BERTHOD, P., HEIL, C., ARANDA, L., "Influence of the morphologic evolution of the eutectic carbides at high temperature on the thermal expansion behavior of refractory cast alloys", Journal of Alloys and Compounds, v. 504, pp. 243-250, 2010. 